\title{
Binary Adaptive Optics
}

\author{
Manuel P. Cagigal*a, Pedro J. Valle ${ }^{\mathrm{a}}$, V. F. Canales ${ }^{\mathrm{a}}$ and M.A. Cagigas ${ }^{\mathrm{b}}$ \\ ${ }^{a}$ Dept. de Física Aplicada, Universidad de Cantabria, Avda. los Castros 48, 39005 Santander, Spain; \\ binstituto Astrofísico de Canarias, Vía Láctea S/N, 38200, La Laguna, Spain
}

\begin{abstract}
In this paper, we introduce the concept of Binary Adaptive Optics (BAO). It is based on the addition of a phase value of $\pi$ in some wavefront points to produce a new compensated wavefront with phase values contained into the $(0, \pi)$ interval. We have checked by computer simulations that the use of BAO is enough for successfully compensating strongly aberrated wavefronts. We used a Point Diffraction Interferometer (PDI) as wavefront sensor. The image obtained from the PDI, once binarized, represents the binary compensating phase map to be sent to the deformable mirror, the spatial light modulator, etc. The main drawback of the BAO system is that the maximum attainable Strehl ratio is 0.5. However, the BAO system has several advantages. The PDI sensor is easy to be manufactured, there is no need of any algorithm to reconstruct the wavefront surface and, finally, its dynamic range is infinite, which is particularly interesting in extremely large telescopes. A detailed analysis of the BAO performances has been carried out by computer simulation.
\end{abstract}

Keywords: adaptive optics, wavefront sensor

\section{INTRODUCTION}

The phase of the wavefront reaching the entrance pupil of an optical system plays a crucial role on the image formation process. Distorted wavefronts provide blurred images and poor image resolution. Adaptive optics systems try to recover the flatness of the distorted wavefront by adding different phase values at particular wavefront points. However, different sources of error prevent the system from reaching its best performance.

The use of binary phase devices in optical systems has been of great interest in different fields. Long focus systems ${ }^{1}$ or system with superresolution ${ }^{2}$ can be achieved by only adding a binary phase plate. Some attempts have also been done to compensate atmospheric aberrations using a static phase plate. In this paper, we propose the use of a simple binary adaptive optics (BAO) system to reach a partial compensation of the wavefront. The goal of the BAO system is to limit the compensated wavefront phase values to the interval $(0-\pi)$. This partial compensation is not as effective as the standard AO since the maximum attainable Strehl is 0.5. In contrast, BAO shows a series of interesting advantages: the wavefront sensor is easy to be manufactured, there is no need of any algorithm to reconstruct the wavefront surface and, finally, its dynamic range is infinite, which is particularly interesting in extremely large telescopes.

The BAO is based on the use of a Point Diffraction Interferometer (PDI) as wavefront sensor. The main drawback of this sensor is it provides wrapped phase values. However, in our application this information is enough to efficiently control the compensating device since it only has to generate binary phases. Hence, BAO systems have several advantages when compared to other sensors. The first one is the PDI sensor is easy to be manufactured. The second one is no algorithm is necessary to reconstruct the wavefront surface. The last one is that its dynamic range is infinite which is particularly interesting in extremely large telescopes. Besides, a large isoplanatic patch is also expected.

The main drawback is it is only able to perform a partial compensation. According to our simulations the attainable Strehl will range from 0.4 to 0.5 . Although this Strehl is small for some extremely demanding applications, like exoplanet detection, it is good enough for most of the telescope uses. 


\section{BINARY ADAPTIVE OPTICS}

The electric field at the image plane can be described as a sum of phasors ${ }^{3}$, which represent the contribution from the different areas at the pupil plane $\mathrm{E}_{x, y}=\mathrm{A}_{x, y} \mathrm{e}^{-\mathrm{i} \phi(x, y)}$. In a plane wavefront the phase value is constant for all the points and it can be taken as $\emptyset_{n}=0$. When this wavefront is focused by a lens, all the phasors are coherently added at the center of the image plane (fig. 1 left). However, in a distorted wavefront the phase $\emptyset_{x, y}$ takes random values on an interval which depends on the distortion magnitude. In this case, the addition of phasors at the center of the image plane tends to zero. (Fig 1 right).

The strategy so far followed by standard adaptive optics (AO) has been to measure the local phase distortion and to use a phase device, usually a deformable mirror, to increase or reduce the local phase of the distorted wavefront forcing all the field phasors to be parallel to the Real axis (Fig 2).

In contrast to this strategy we propose here to increase the phase in a magnitude of $\pi$ in those wavefront areas where the corresponding phasor is under the Imaginary axis. In such a case, the imaginary component of the field is close to zero whilst the real component of the field will take a significant value.

In the AO technique one can expect to reach a maximum Strehl ratio of 1, although current systems hardly overpass the value of 0.5 . Since the maximum attainable Strehl by BAO is 0.5 it could seem that this approach is not as effective as the standard one. However there is a series of interesting advantages along the different steps of the BAO compensation process which make it really interesting.

\section{ANALYSIS OF THE COMPENSATION PROCESS}

In this section we will stablish the different steps involved in the BAO process.

\subsection{Wavefront sensing}

First, it is interesting to state that the BAO does not require a wavefront sensing as accurate as that of the standard AO. In this case we only need to know if the local wavefront phase is in the interval $(0, \pi)$ or in the interval $(0,-\pi)$. This task can be carried out by a simple Point Diffraction Interferometer (PDI). The PDI is a common-path interferometer that has the advantage of being simple, cheap and robust ${ }^{4}$.

However, in spite of its simplicity, this sensor is not widely used because it is difficult to recover the actual values of the wavefront local phase, except in some particular cases. Nevertheless, this sensor is appropriate for BAO since it allows a direct recovery of the phase information needed. The PDI sensor scheme is shown in Figure 3 . The distorted wavefront located on the entrance pupil of the optical system at a distance equal to the focal length of the lens L1 is focused by lens L1 in a mask (M). The mask has a small central area with transmittance or phase different from the rest of the mask. The central area creates a reference wavefront which interferes with the rest of the wavefront. The resulting interference pattern is detected by a camera placed at a plane which is conjugated to the pupil plane.

\subsection{Phase retrieval algorithm}

In general, the second step when using an $\mathrm{AO}$ system is to retrieve the phase map of the distorted wavefront from the information provided by the sensor. In the BAO, the intensity at the camera plane of Fig. 3 can be described by:

$$
I_{x, y}=\left|A_{x, y} e^{-i \phi_{x, y}}+A_{0} e^{-i \phi_{0}}\right|^{2}
$$


where $\mathrm{A}_{x, y} \mathrm{e}^{-\mathrm{i} \phi_{x, y}}$ is the field coming from the incident wavefront and $\mathrm{A}_{0} \mathrm{e}^{-\mathrm{i} \phi_{0}}$ is the reference field. From Eq.(1) it is easy to obtain:

$$
\cos \left(\phi_{\mathrm{x}, \mathrm{y}}-\phi_{0}\right)=\frac{I_{\mathrm{x}, \mathrm{y}}}{2\left|A_{\mathrm{x}, \mathrm{y}}\right|^{2}}-1
$$

In those points where the phase difference is between 0 and $\pi$ Eq. (2) takes a positive value and it is negative when the phase difference is between 0 and $-\pi$. The binary mask $(B M)$ obtained from Eq. 2 will be:

$$
B M_{x, y}=\left\{\begin{array}{lll}
0 & \text { if } & \cos \left(\phi_{\mathrm{x}, \mathrm{y}}-\phi_{0}\right) \geq 0 \\
\pi & \text { if } & \cos \left(\phi_{\mathrm{x}, \mathrm{y}}-\phi_{0}\right)<0
\end{array}\right.
$$

From the binary mask $B M_{(x, y)}$ we obtain the sign map:

$$
S M_{x, y}=\left\{\begin{array}{l}
1 \quad \text { if } \quad B M_{x, y}=0 \\
-1 \quad \text { if } \quad B M_{x, y}=\pi
\end{array}\right.
$$

Figure 4 shows a comparison between the binary map obtained from a direct binarization of the incoming distorted wavefront (left) and that obtained using Eq.(3). It can be seen that, although they are not identical, there exists a great similitude between both phase maps. The binary phase map retrieved from the detected image, once applied the algorithm of eq.(3), is good enough to produce a compensated wavefront. It can be seen that the retrieving algorithm is simple and fast.

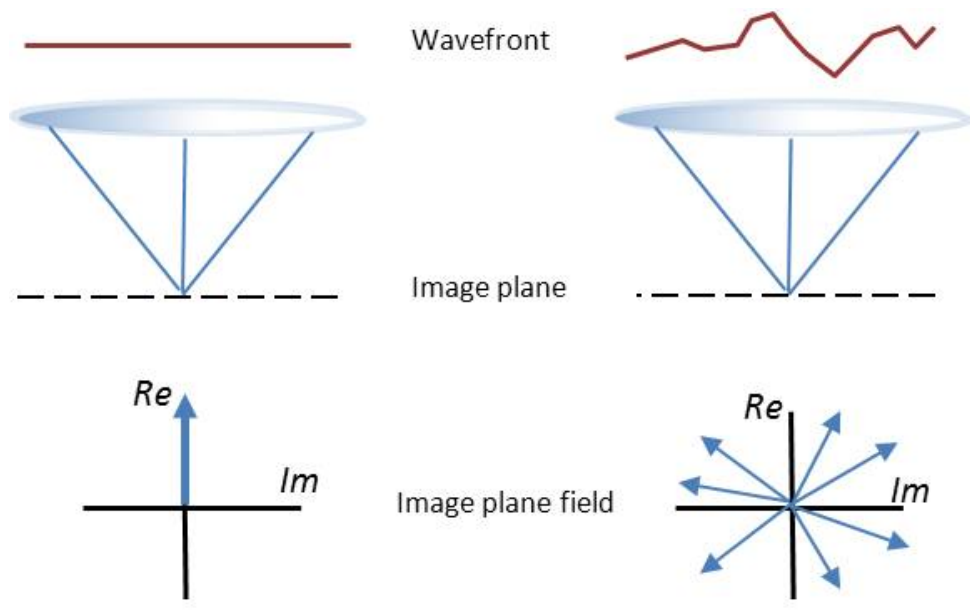

Figure 1. Example of plane wavefront (left), where all the field phasors contribute constructively to the final field at the image plane. For a distorted wavefront (right) the random phase distribution causes a small or even null total field. 

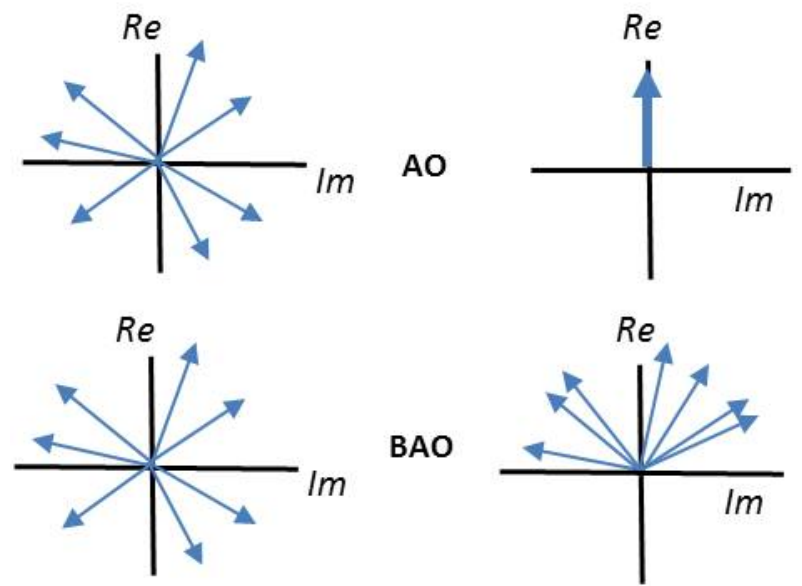

Figure 2. Phasors with a random phase distribution (left). The addition of these phasors tends to zero. The same set of phasors but some of them have been rotated with respect to the horizontal axis to convert the negative vertical component into positive. In this case the total field imaginary component tends to zero whilst the real component tends to a non-zero value.

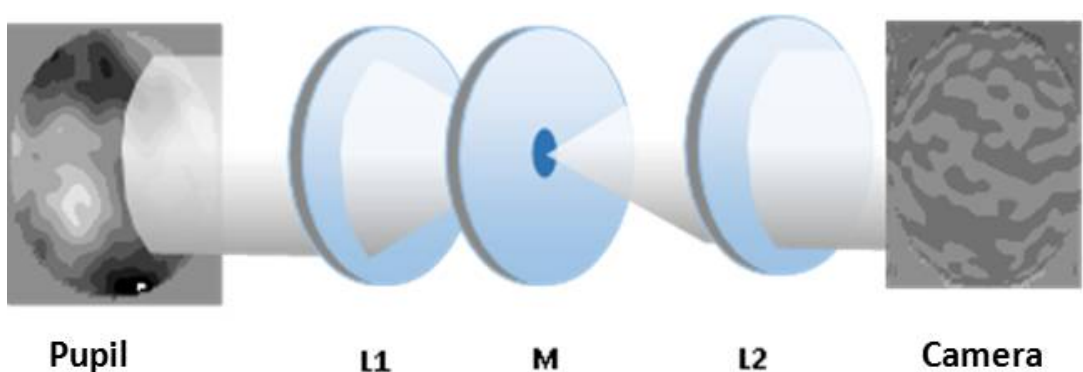

Figure 3. The PDI sensor consists on a 4-f system, formed by lenses L1 and L2, and a mask placed in the common focal plane (M). The interference pattern produced by the mask is recorded on a camera placed after the lens L2.
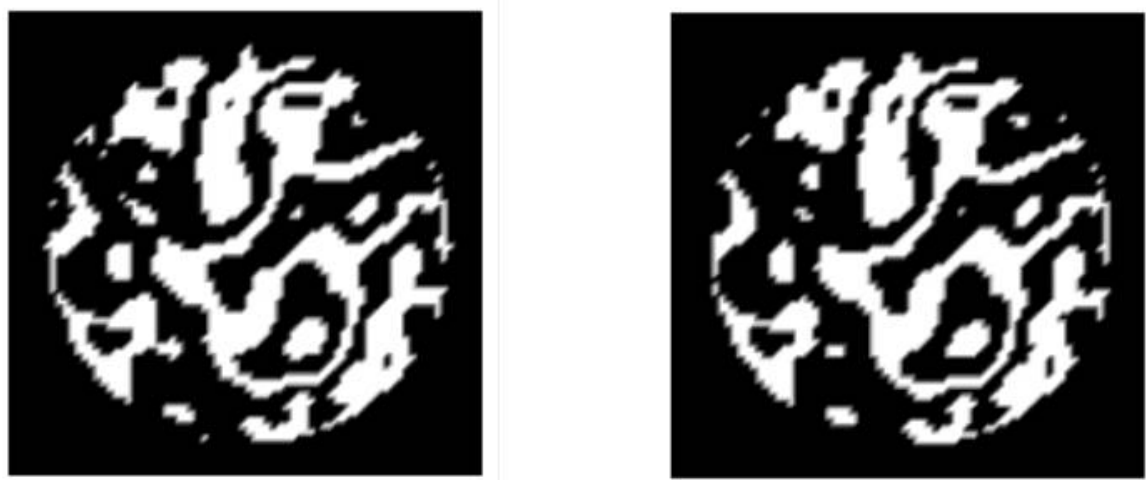

Figure 4. Comparison between the direct binarization of the wavefront defined on the entrance pupil of the optical system (left) and the binarized wavefront resulting from the PDI sensor. The difference between both images is almost negligible. 


\subsection{Phase compensation}

The phase compensation process of the incoming distorted field $A_{x, y} \mathrm{e}^{-i \phi x, y}$ is based on the sign map provided by the PDI and given by Eq.(4). Hence, it is enough to multiply the distorted field by the sign map $S_{x, y}$. The resulting compensated field will be:

$$
C F_{x, y}=S M_{x, y} A_{x, y} \mathrm{e}^{-\mathrm{i} \phi_{x, y}}
$$

The BAO only requires a system able to produce binary phase values as those shown in Fig (4). This can be carried out by a deformable mirror (DM) or by a spatial light modulator (SLM). This compensation process has different advantages. First, the image retrieved from the sensor can be directly sent to the compensator without any further processing. Hence the time delay between sensing and compensation is really short and no extra errors are introduced during the wavefront reconstruction process. Second, it is quite easy to control a compensator device (DM or SLM) when it only provides binary values. Similar approaches can be found in several sources ${ }^{5,6}$.

In general one can say that, although the maximum Strehl attainable for the BAO is only a half of that of SAO, the low requirements for the wavefront sensor and compensator, the simplicity of the phase retrieval algorithm and the short delay between sensing and compensation, make $\mathrm{BAO}$ a competitive alternative to the SAO scheme.

\section{BAO PERFORMANCE ANALYSIS}

The analysis of the BAO performance was carried out by computer simulation. A series of phase screens $\phi_{x, y}$ following the atmospheric statistics were simulated. The corresponding electric field was calculated $A \mathrm{e}^{-i \phi x, y}$. After that, we always considered the amplitude $A$ constant and equal to 1 . A wavefront sensor like that of Figure 3 was simulated taking advantage of the Fast Fourier Transform (FFT). We used two kinds of mask, those with constant phase and a different transmittance in the central circle and those with constant transmittance and a difference in phase of $\pi$ in the central circle. Both of them worked properly, although the image provided by the last one presented a better signal-to-noise ratio. The image of the sensor camera was used to obtain the phase map and we performed the compensation as shown by Eq.(5). Then, the resulting $C F_{x, y}$, was multiplied by the system entrance pupil and Fourier transformed to obtain the compensated image.

\subsection{Effect of the PDI mask size}

Since the reference field in the PDI is taken from the distorted PSF, it is interesting to know if the sensor, and consequently the BAO system efficiency are affected by the degree of wavefront distortion. We simulated a series of wavefronts for different $D / r_{0}$ values, where $D$ is the diameter of the optical system pupil and $r_{0}$ is the Fried parameter. The larger $D / r_{0}$ value the worst the atmosphere is.

The result of this analysis is shown in figure 5 . We can see that for $D / r_{0}=0$, that is, for no distortion, the Strehl takes a value of 1 . It drops quickly, as expected, so that for $D / r_{0}=5$ it takes a value slightly under 0.5 . When the $D / r_{0}$ value increases, the Strehl decreases slowly to reach a minimum value of 0.4 .

Hence, we can say that the BAO technique is basically unaffected by atmospheric conditions. Furthermore, although the maximum Strehl is only attainable for $D / r_{0} \approx 4$, it keeps a high value $(>0.4)$ for higher $D / r_{0}$ values.

On the other hand, fig. 5 also shows different curves obtained for a series of PDI masks with different sizes. We have taken as a reference the size of the PSF generated by lens L1 in fig. 3. In fig. 5, the mask size ranges from one fourth of the PSF size up to the PSF size. The difference in result is almost negligible, taking into account that the whole process is affected by atmosphere distortion. We can then conclude that the mask size is not a key parameter and errors in the mask manufacture will not affect the wavefront phase estimate provided by the wavefront sensor. 


\subsection{Effect of the number of actuators}

Given that the size of the Fried parameter corresponds to an area in which the phase variation is smaller than one radian and that the BAO only acts in those areas in which the difference in phase is larger than $\pi$ radians it seems that the numbers of actuators required for the compensating device has to be low.

Fig 6 shows the Strehl value attainable by the BAO system as a function of the actuator size on the compensating device. The analysis is repeated for three different $D / r_{0}$ values. It can be seen that a good compensation can be achieved even when the number of actuators in the compensating device is low. In particular, for $D / r_{0}=10$ the maximum possible Strehl is achieved for an actuator size of $r_{0} / 2$ whilst for $D / r_{0}=40$ it is enough an actuator size of $r_{0} / 5$ for achieving the maximum possible Strehl. This result is particularly interesting for extremely large telescopes where the $D / r_{0}$ value is high and a low sampling of the $r_{0}$ distance becomes really important.
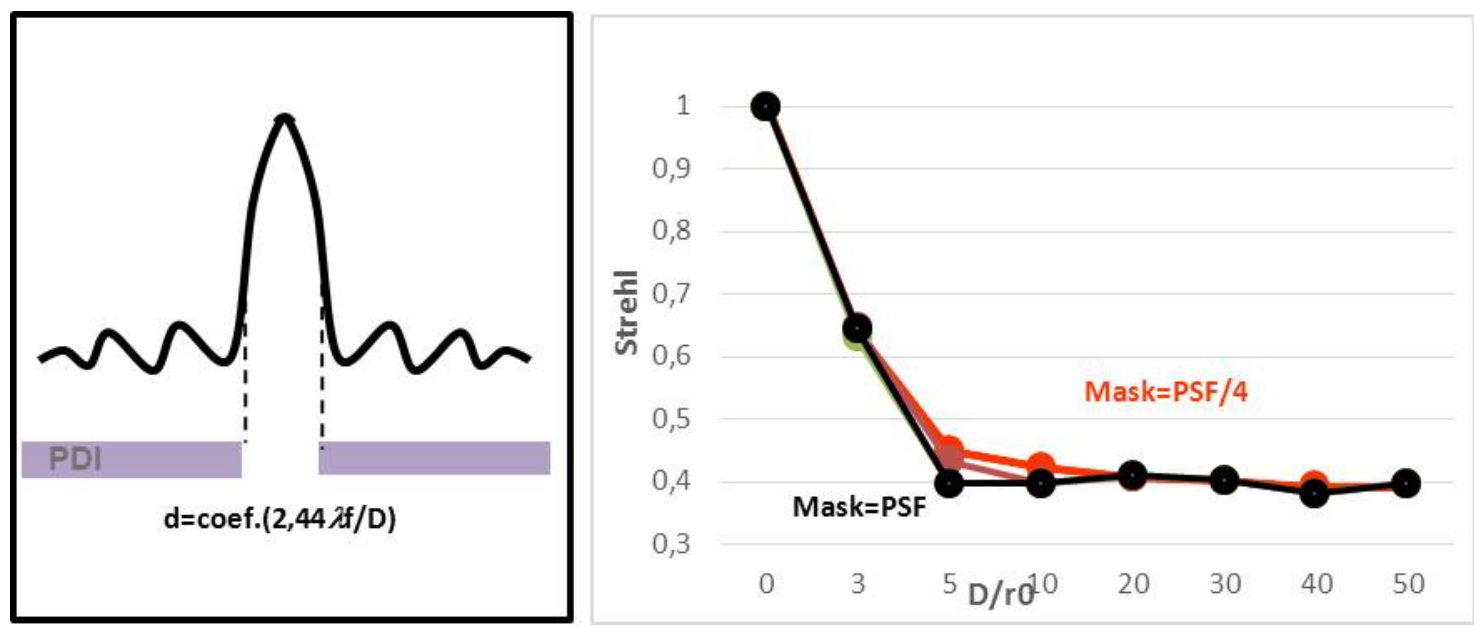

Figure 5. Left: PDI mask with a central hole with size proportional to the PSF size. Right: Strehl attained by the BAO system as a function of $D / r_{0}$ for different PDI mask sizes.
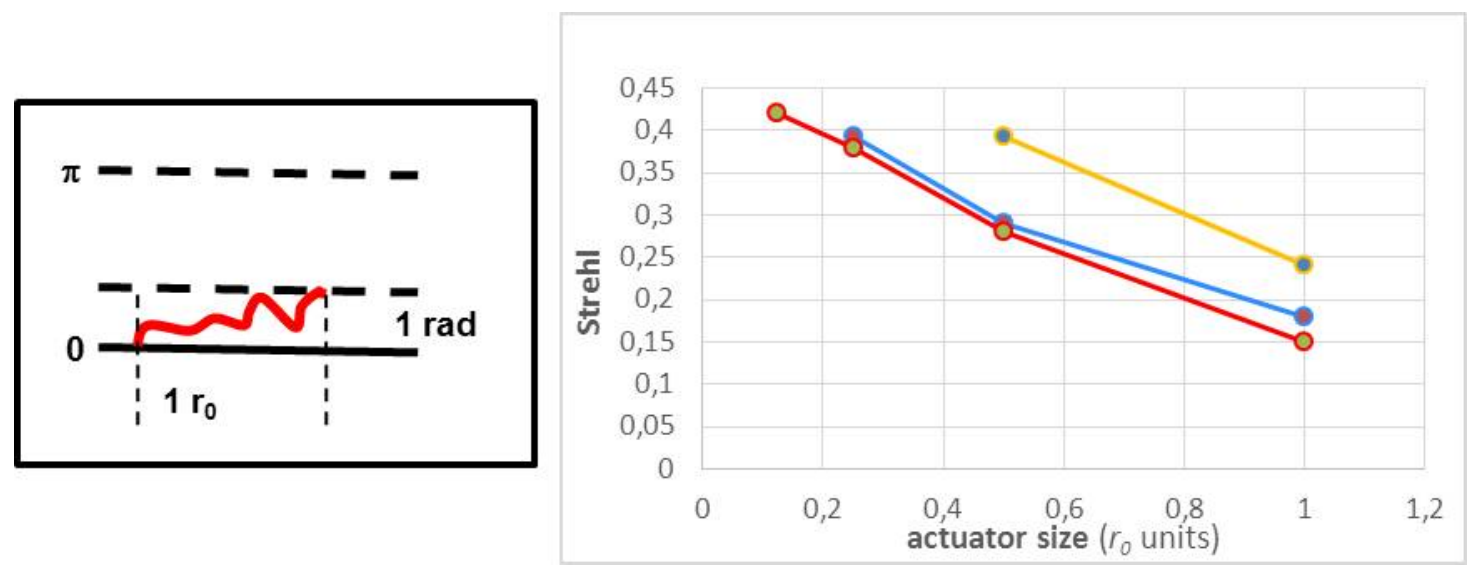

Figure 6. Left: definition of the Fried parameter. Right: Strehl attained by the BAO system as a function of the number of actuators for different $D / r_{0}$ values. 


\subsection{Isoplanatic patch}

The angular behavior of the BAO system was also checked. Figure 7 compares how the compensation decreases as a function of the angular separation between the guide star and the companion. This angular distance is given by $\Omega \square=$ $0.62 r_{0} / h$, where $h$ is the distance between the target and the telescope.

Figure 7 shows that the ideal AO system performs better than the BAO system, but the maximum Strehl ratio drops quickly to similar values for both ideal $\mathrm{AO}$ and $\mathrm{BAO}$ when the angular distance $\Omega$ increases. This analysis was repeated for different $D / r_{0}$ values. Hence, we can expect the BAO system to present isoplanatic patch sizes comparable to those of the ideal AO system.
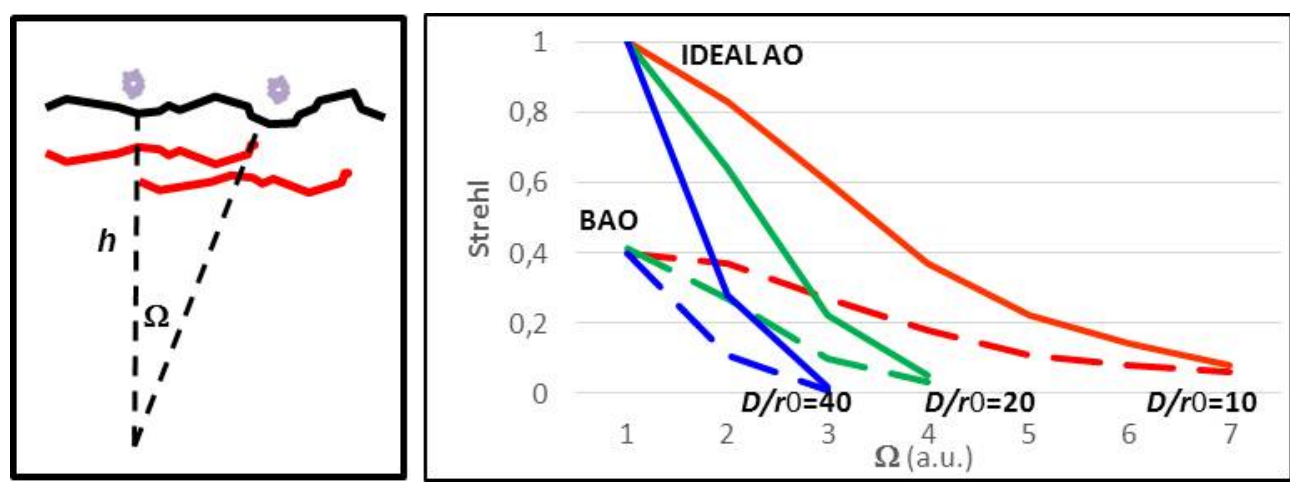

Figure 7. Left: Two targets with angular separation $\Omega$. Right: Strehl attained by the ideal AO and BAO systems as a function of the angular separation for different $D / r_{0}$ values.

\section{CONCLUSIONS}

Table 1 shows a comparison between the different steps of AO and the corresponding ones for BAO. We can see on that, in spite of the maximum Strehl ratio allowed for BAO is 0.5 , it presents other features that make of it an advantageous alternative to traditional $\mathrm{AO}$ systems. In particular the absence of wavefront reconstruction, which implies a negligible time delay between sensing and compensation, and the infinite dynamic range of the sensor and compensator make the BAO interesting for very large telescopes.

Table 1. Comparison between the different steps of AO and the corresponding ones for BAO.

\begin{tabular}{|c|c|c|}
\hline & AO & BAO \\
\hline WFS & $\begin{array}{l}\text { - Slopes/curvatures estimates } \\
\text { - Low Sampling Rate } \\
\text { - Low Dynamic Range }\end{array}$ & $\begin{array}{l}\text { - Direct phase estimates } \\
\text { - High Sampling Rate } \\
\text { - Infinite Dynamic Range }\end{array}$ \\
\hline Control & $\begin{array}{l}\text { - Wf shape estimate from Zernikes } \\
\text { - Time delay }\end{array}$ & $\begin{array}{l}\text { - Only thresholding. No need } \\
\text { of Zernikes } \\
\text { - Short time delay }\end{array}$ \\
\hline Deformable mirror & $\begin{array}{l}\text { - Needs high accuracy } \\
\text { - Needs high sampling rate } \\
\text { - Limited Dynamic Range }\end{array}$ & $\begin{array}{l}\text { - Binary values }(0-\pi) \\
\text { - Low sampling rate: } \\
\quad 1 \text { actuator/r0 } \\
\text { - Infinite dynamic range }\end{array}$ \\
\hline
\end{tabular}




\section{ACKNOWLEDGMENTS}

We acknowledge the financial support given by the Ministerio de Economía y Competitividad via project AYA201678773-C2-1-P

\section{REFERENCES}

[1] de Juana D.M., Canales V.F., Valle P.J. and Cagigal M.P. " Focusing properties of annular binary phase filters," Optics Communications, 229, 71-77 (2004).

[2] Canales V.F., Valle P.J.and Cagigal M.P., " Analysis of Strehl ratio limit with superresolution binary phase filters," Chin. Opt. Lett. 14, 071101 (2016)

[3] Goodman J.W., " Statistical properties of laser speckle patterns," in Laser speckle and related phenomena (J.C.Dainty ed.) Springer-Verlag, Berlín, Topics in Applied Physics 9, 9 (1984)

[4] Crabtree P., Woods C.L., Khoury J. and Goda M., " Binary phase-only filtering for turbulence compensation in fiber-coupled free-space laser communication systems," Appl. Opt.. 46, 1 (2007)

[5] Kim H.M., Jeong J.W., Kwak C.H., and Lee S.S, " Binary phase spatial modulation using photoinduced anisotropy in amorphous As2S3 thin film,," S Appl. Opt.. 34, 10 (1995).

[6] Acosta E., Chamadoira S. and Blendowske R., " Modified point diffraction interferometer for inspection and evaluation of ophthalmic components," J. Opt. Soc. Am. A. 23, 3 (2006) 